\title{
Metabolic profiling of maize seeds with both insect- and herbicide-resistance genes (cry1Ab and epsps), dual herbicide-resistance genes (epsps and pat), and natural genotypic varieties
}

Weixiao Liu* ${ }^{*}$, Mei Dong, Lixia Meng, Yusong Wan and Wujun Jin*

\begin{abstract}
Background: Widely targeted metabolomics was applied to estimate the differences in the metabolite profiles of maize seeds from 3 natural genotypic varieties and 4 genetically modified (GM) lines.

Results: Pairwise comparison with their isogenic controls revealed 71, 121, 43 and 95 differentially accumulated metabolites (DAMs) in GM maize seeds of C0030.2.4, C0030.3.5, C0010.1.1 and C0010.3.1, respectively. KEGG pathway enrichment analysis showed that most of these DAMs participated in the biosynthesis of secondary metabolites and purine metabolism in GM maize C0030.2.4 and C0030.3.5, but participated in tryptophan metabolism and 2-oxocarboxylic acid metabolism in C0010.3.1 seeds and in metabolic pathways and the biosynthesis of secondary metabolites in C0010.1.1 seeds. The data also showed that the differences in metabolite accumulation, both total DAMs and co-DAMs, among the different natural genotypic varieties (418 DAMs and 39 co-DAMs) were greater than those caused by genetic modification (330 DAMs and 3 co-DAMs).
\end{abstract}

Conclusions: None of the DAMs were identified as new or unintended, showing only changes in abundance in the studied maize seeds. The metabolite profile differences among the 3 non-GM lines were more notable than those among GM lines. Different genetic backgrounds affect metabolite profiling more than gene modification itself.

Keywords: Widely targeted metabolomics, Differentially accumulated metabolites, Unintended effects, Genetic modification, Natural genotypic varieties, Maize seeds

\section{Introduction}

The commercialization of genetically modified (GM) crops has not only led to considerable economic gains but also reduced environmental problems. However, due to unintended effects, GM crops remain a focus of debate $[1,2]$. Therefore, the overall safety of GM crops must be evaluated [3-6]. Omics (e.g., genomics, transcriptomics,

\footnotetext{
*Correspondence: liuweixiao@caas.cn; jinwujun@caas.cn Biotechnology Research Institute, Chinese Academy of Agricultural Sciences, No. 12 Zhongguancun South St., Haidian District, Beijing 100081, China
}

proteomics, and metabolomics) methods developed in recent years enable unbiased safety assessments and provide comprehensive and detailed insights into unintended changes in studied GM crops [7, 8]. Compared to genomics and transcriptomics, proteomics and metabolomics can be used to better evaluate the levels of nutrients and other food quality and safety factors [9]. Therefore, isobaric tags with relative and absolute quantitation (iTRAQ)-based proteomics have been widely used in the safety evaluation of GM crops [10-15]. Our previous study also showed that this method can successfully 
assess unintended changes in GM maize [16]. In addition to proteins, metabolites not only influence crop growth, development and reproduction [17, 18], but also largely affect crop quality traits [19]. Recently, advances in metabolite profiling technology have made it possible to comprehensively compare metabolite differences in crops affected by growth environments [9], genetic engineering and conventional cross-breeding [20-24]. A recent study has even shown that 10 times more DAMs were observed under different growing environments [25].

Insect resistance and herbicide resistance are the main features of GM crops. The Bacillus thuringiensis (Bt) cry gene (encoding the Cry protein exhibiting high selectivity and a broad insecticidal spectrum) has been widely used in GM crops [26]. Among herbicide-resistance genes, the epsps (encoding 5-enolpyruvate shikimic acid-3-phosphate synthase, EPSPS) [27-29] and bar/pat (encoding phosphinothricin acetyl transferase, PAT) [30, 31] genes are most widely used.

Recently, published data have analysed the effects of HIR (herbicide- and insect-resistance) gene cassettes and different growing environments on the transgenic maize metabolome [25]. In this study, widely targeted metabolomics was used for the analysis of maize seeds from 4 GM maize lines, with two carrying both insect- and herbicide-resistance genes (C0030.2.4 and C0030.3.5) and two with dual insertion of herbicide-resistance genes (C0010.3.1 and C0010.1.1), and 3 non-GM maize lines (NH101, DH605 and NH106) to fill the knowledge gaps concerning metabolite differences in GM and natural genotypic varieties and enrich the field of GM and nonGM maize metabolomics.

\section{Materials and methods Plant materials}

Seeds of the GM maize lines C0030.2.4 (A1; carrying epsps and cry1Ab genes), C0010.3.1 (A2; carrying epsps and pat genes), C0030.3.5 (B1; carrying epsps and cry $1 A b$ genes) and C0010.1.1 (C2; carrying epsps and pat genes) and the corresponding non-GM isogenic varieties NH101 (A0), DH605 (B0) and NH106 (C0) were selected for study. The details of these maize lines were described previously [16]. Maize seeds with full grains and uniform sizes were provided by Dabeinong Co. The GM maize lines and their non-GM parents were collected from the same natural growth environment and stored at $-80^{\circ} \mathrm{C}$.

\section{Metabolite preparation}

Six biological replicates of seeds of the 7 studied maize lines were used for metabolite profiling. Maize seed grains of each line were ground in liquid $\mathrm{N}_{2}$. The total metabolites were extracted from $100 \mathrm{mg}$ of seed powder with $70 \%$ aqueous methanol overnight at $4{ }^{\circ} \mathrm{C}$. Following centrifugation at $10,000 \mathrm{~g}$ for $10 \mathrm{~min}$, the extracts were absorbed by a CNWBOND Carbon-GCB SPE Cartridge (ANPEL, Shanghai, China) and filtered before UPLC (ultra-performance liquid chromatography)-MS/MS (mass spectrum) analysis.

\section{UPLC conditions and ESI-QTRAP-MS/MS}

The extracted metabolites were analysed using a UPLCESI-MS/MS system (UPLC, Shim-pack UFLC Shimadzu CBM30A system; MS, Applied Biosystems 4500 Q TRAP). The analytical conditions and ESI-QTRAP-MS/ MS operating parameters were described previously [32].

\section{Metabolite data analysis}

Quality control (QC) analysis was conducted to confirm the reliability of the data. A QC sample was prepared by mixing sample extracts and inserted into every two samples to monitor changes over repeated analyses. Orthogonal partial least squares-discriminant analysis (OPLS-DA) was used to maximize the metabolome differences by removing irrelevant differences between the GM and non-GM parent samples. Based on the OPLSDA results, the metabolites of different maize samples were preliminarily screened using the variable importance in projection (VIP) values of the obtained multivariate OPLS-DA model. The P-value and fold change values of the univariate analysis were combined to further screen DAMs. Metabolites with a VIP $\geq 1$ and a fold change $\geq 2$ or $\leq 0.5$ were considered differential metabolites for group discrimination [33]. Principal component analysis (PCA) was performed using the statistics function prcomp within $\mathrm{R}$ (www.r-project.org). A heatmap based on hierarchical cluster analysis was generated in R software (www.r-project.org). Kyoto Encyclopedia of Genes and Genomes (KEGG) pathway annotation of the DAMs was carried out using the KEGG database http:// www.genome.jp/kegg/ [34, 35].

\section{Results \\ Metabolite profiling of maize seeds}

In total, 664 metabolites were successfully detected in maize seeds by widely targeted metabolomics (Additional file 1: Table S1). The detected metabolites were diverse and could be classified into 31 classes, including amino acids and derivatives, nucleotides and derivatives, phenolic acids, organic acids, sugar alcohols, etc. PCA yielded PC1 (30.1\%) and PC2 (15.69\%) as the two main components. Repeated samples of the 7 maize lines clustered together well (Fig. 1A). Cluster analysis of the identified metabolites showed that the metabolite profiles of the 7 maize lines were divided into two groups as follows: $\mathrm{B} 0, \mathrm{~A} 1, \mathrm{~A} 0$, and $\mathrm{B} 1$ and $\mathrm{A} 2, \mathrm{C} 2$, and $\mathrm{C} 0$. The metabolite profiles of $\mathrm{A} 0$ and $\mathrm{B} 1$ shared the highest similarity among 
A

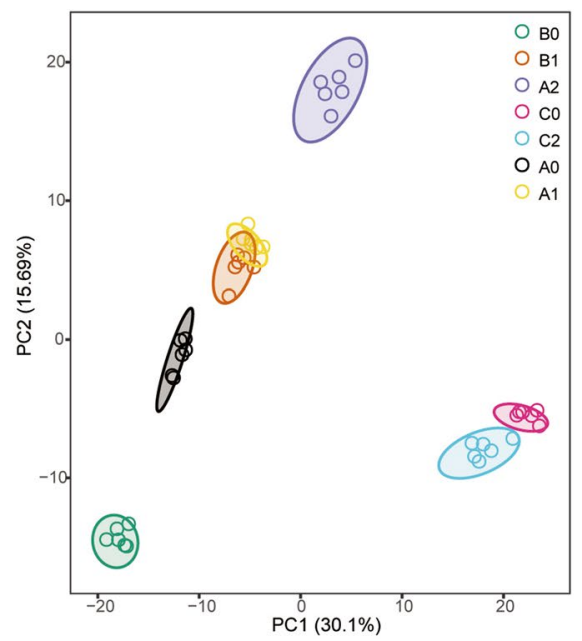

B

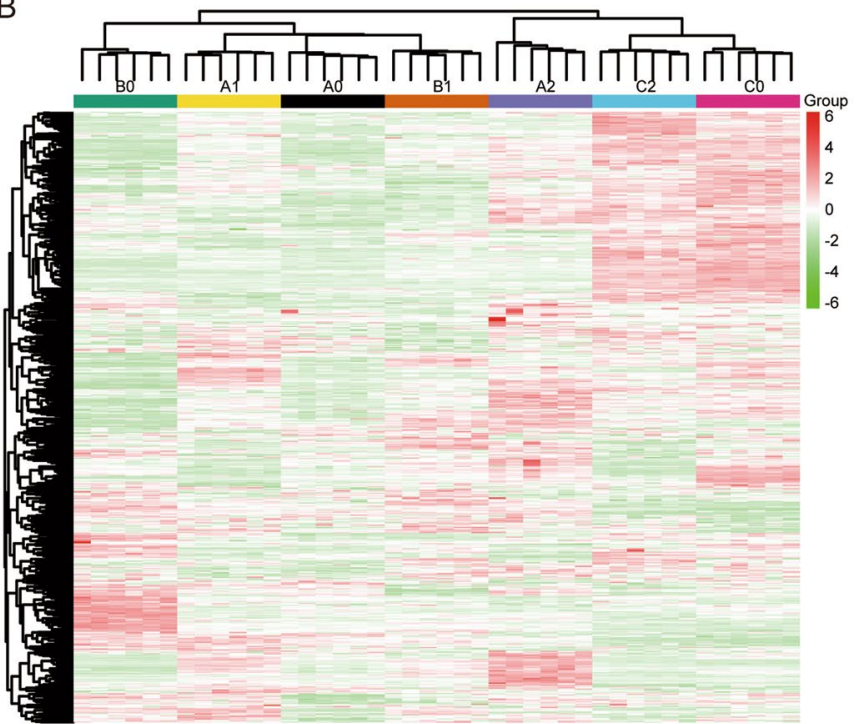

Fig. 1 Metabolite abundance pattern analysis. A Principal component (PC) analyses of metabolite levels in seeds of 7 maize lines. Score plot of the first two PCs with the explained variance. B Hierarchical clustering of 7 maize lines using metabolite accumulation data. In the heatmap, every repeat of each maize line is visualized in a single column. Metabolite accumulation is shown in different colours, where red indicates high abundance and green indicates low relative expression

the first group and shared higher similarity with those of $\mathrm{A} 1$ and $\mathrm{A} 0$ than those of $\mathrm{B} 1$ and B0. The metabolite profiles of $\mathrm{C} 2$ and $\mathrm{C} 0$ shared higher similarity than those of $\mathrm{A} 2$ and $\mathrm{C} 0$ did (Fig. 1B).

\section{Identification of DAMs and co-DAMs in maize seeds}

Metabolites with changes in abundance greater than 2.0 -fold or lower than 0.5 -fold (VIP $\geq 1$ ) were identified as DAMs. The numbers of DAMs in the different comparison groups are summarized in Table 1. There were 71 DAMs identified in the A1/A0 sample, including 59 upregulated metabolites and 12 downregulated metabolites (Additional file 1: Table S2). One hundred twenty-one DAMs were identified by the comparison of B1 with B0, of which 86 were upregulated and 35 were downregulated (Additional file 1: Table S3). A total of 95 DAMs were identified in the A2/A0 sample, including 88 upregulated metabolites and 7 downregulated metabolites (Additional file 1: Table S4). There were 43 DAMs identified in the $\mathrm{C} 2 / \mathrm{C} 0$ sample, including 12 upregulated metabolites and 31 downregulated metabolites (Additional file 1: Table S5). In total, 330 DAMs were identified in four GM/non-GM comparison groups (Table 1). There were 112, 169 and 137 DAMs identified in the $\mathrm{A} 0 / \mathrm{B} 0, \mathrm{~B} 0 / \mathrm{CO}$ and $\mathrm{A} 0 / \mathrm{C} 0$ samples, respectively (Additional file 1: Tables S6-8). In total, 418 DAMs were identified in three non-GM/non-GM comparison groups (Table 1). Pairwise comparisons revealed that there were $9,18,10$ and 2 new metabolites in $\mathrm{A} 1 / \mathrm{A} 0, \mathrm{~B} 1 / \mathrm{B} 0, \mathrm{~A} 2 / \mathrm{A} 0$ and $\mathrm{C} 2 / \mathrm{C} 0$, respectively

Table 1 Summary of the numbers of DAMs in different comparison groups

\begin{tabular}{|c|c|c|c|c|}
\hline $\begin{array}{c}\text { Comparison } \\
\text { groups }\end{array}$ & $\begin{array}{c}\text { No. of upregulated } \\
\text { metabolites }\end{array}$ & $\begin{array}{c}\text { No. of downregulated } \\
\text { metabolites }\end{array}$ & No. of DAMs & $\begin{array}{c}\text { Total no. of } \\
\text { DAMs }\end{array}$ \\
\hline A1/A0 & 59 & 12 & 71 & \\
\hline B1/B0 & 86 & 35 & 121 & 330 \\
\hline C2/C0 & 12 & 31 & 43 & \\
\hline A2/A0 & 88 & 7 & 95 & \\
\hline A0/B0 & 58 & 54 & 112 & \\
\hline B0/C0 & 39 & 130 & 137 & \\
\hline A0/C0 & 19 & 118 & & \\
\hline
\end{tabular}



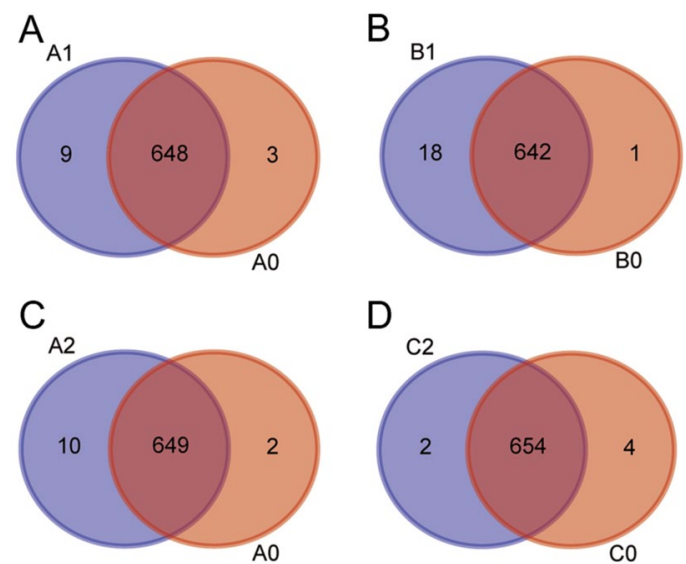

Fig. 2 Pairwise comparisons of differentially accumulated metabolites between GM/non-GM maize seeds. A A1/A0, B B1/B0, C A2/A0 and D C2/CO

(Fig. 2A-D). These new metabolites were new only to their non-GM parents, but were present in other nonGM maize lines (Additional file 1: Figure S1).

\section{Analysis of the identified DAMs of $A 1 / A 0$ and $B 1 /$ $B 0$ resulting from the insertion of both insect- and herbicide-resistance genes (cry $1 A b$ and epsps)}

KEGG pathway analysis showed that the DAMs of A1/ A0 were annotated to 22 KEGG pathways. Biosynthesis of secondary metabolites (ko01110) and purine metabolism (ko00230) were the primary enriched pathways, followed by flavone and flavonol biosynthesis (ko00944) (Fig. 3A). The DAMs of B1/B0 were annotated to 38 KEGG pathways. Biosynthesis of secondary metabolites (ko01110) and purine metabolism (ko00230) were the primary enriched pathways, followed by pentose and glucuronate interconversions (ko00040) (Fig. 3B).

\section{Analysis of the identified DAMs of A2/A0 and C2/C0 resulting from the dual insertion of herbicide-resistance genes (epsps and pat)}

KEGG pathway analysis showed that the DAMs of A2/ A0 were annotated to 45 KEGG pathways. Tryptophan metabolism (ko00380) and 2-oxocarboxylic acid metabolism (ko01210) were the primary enriched pathways, followed by tyrosine metabolism (ko00350) (Fig. 4A). The DAMs of C2/C0 were annotated to 18 KEGG pathways. Metabolic pathways (ko01100) and biosynthesis of secondary metabolites (ko01110) were the primary enriched pathways, followed by valine, leucine and isoleucine biosynthesis (ko00290) (Fig. 4B).
co-DAMs identified in seeds of all four GM and three non-GM maize lines

Among the identified DAMs, Lmhp003013, pme3961 and Lmyp004081 were simultaneously identified in four comparison groups, $\mathrm{A} 1 / \mathrm{A} 0, \mathrm{~B} 1 / \mathrm{B} 0, \mathrm{~A} 2 / \mathrm{A} 0$ and $\mathrm{C} 2 / \mathrm{C} 0$, and designated co-DAMs (Fig. 5A). Only pme3961 was involved in purine metabolism and metabolic pathways (Table 2). The regulatory trends of these co-DAMs are shown in Fig. 5B. These co-DAMs were downregulated in GM maize line $\mathrm{C} 2$ seeds but upregulated in GM maize line A1 and B1 seeds. Lmhp003013 and Lmyp004081 were upregulated in seeds of the GM maize line A2, but pme3961 was downregulated. In the non-GM maize comparison groups $\mathrm{A} 0 / \mathrm{B} 0, \mathrm{~B} 0 / \mathrm{CO}$ and $\mathrm{A0} / \mathrm{C} 0,39$ coDAMs were identified (Fig. 5C). Their regulatory trends are shown in Fig. 5D. pme3961 was also identified as a co-DAM in three non-GM maize comparison groups.

\section{Integrated proteomics and metabolomics analyses}

Integrated proteomics and metabolomics analyses showed that DEPs [16] were involved in $61,33,72$ and 72 KEGG pathways in the A1/A0, B1/B0, A2/A0 and C2/ $\mathrm{C} 0$ comparison groups. DAMs were involved in $24,38,45$ and 18 KEGG pathways in the A1/A0, B1/B0, A2/A0 and $\mathrm{C} 2 / \mathrm{C} 0$ comparison groups. These identified DEPs and DAMs participated in 14, 14, 31 and 13 common KEGG (co-KEGG) pathways in the four comparison groups (Table 3). The analyses also showed that only the DEPs and DAMs in GM maize A2 seeds were simultaneously annotated in phenylalanine, tyrosine and tryptophan biosynthesis (Ko00400). However, the introduction of exogenous EPSPS did not affect the expression levels of six other enzymes, or cause an increase in abundance of seven metabolites (Additional file 1: Figure S2).

\section{Discussion}

Safety assessments of GM crops are performed to demonstrate that these crops are substantive equivalents to their non-GM isogenic controls to ensure consumer health and safety. Therefore, nontargeted methods of substantive equivalence analysis are the best ways to evaluate the safety of GM crops. Metabolites accumulate in seeds, largely affecting crop quality traits [19]. Here, metabolite profiling of maize seeds was performed by widely targeted metabolomics to facilitate the study of alterations in metabolite accumulation regulated by transgenic modifications and/or different natural genotypic variations. Four GM maize lines were used to study the effects of genetic modification with the same and/or different genetic backgrounds, while three natural genotypic maize varieties were studied to understand the metabolite differences among 


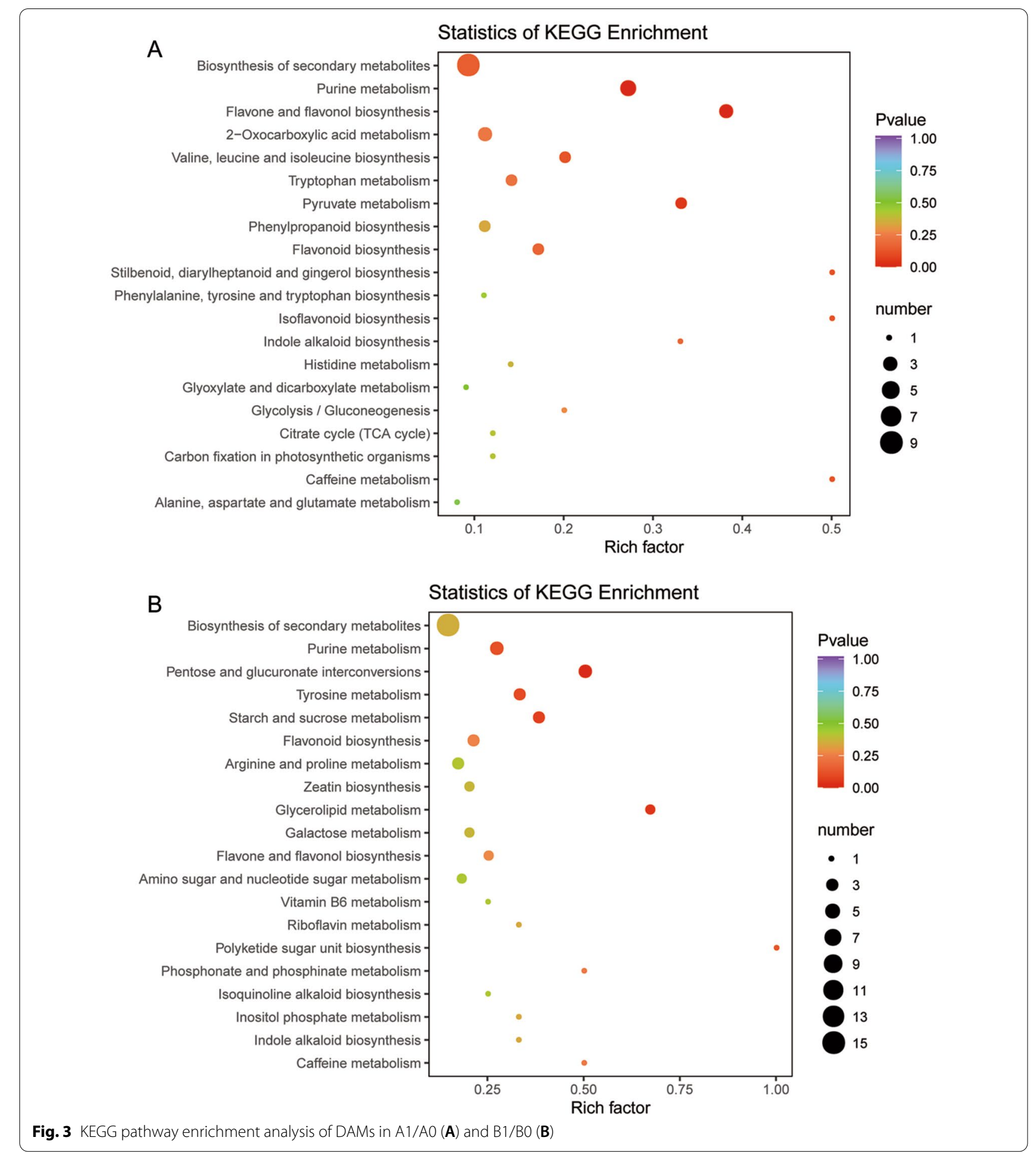

different genetic variations. In this study, 664 metabolites were identified from seeds of 7 maize lines. There were $71,121,43$ and 95 DAMs in A1, B1, C2 and A2 GM maize seeds, respectively. These DAMs were not identified as new metabolites and showed only changes in abundance. These results are consistent with results previously reported for other GM crops [22].

There were 112, 169 and 137 DAMs identified by comparison of the non-GM maize lines $\mathrm{B} 0 / \mathrm{A} 0, \mathrm{~B} 0 / \mathrm{C} 0$ and $\mathrm{A} 0 / \mathrm{C} 0$, respectively. The differences in metabolite 


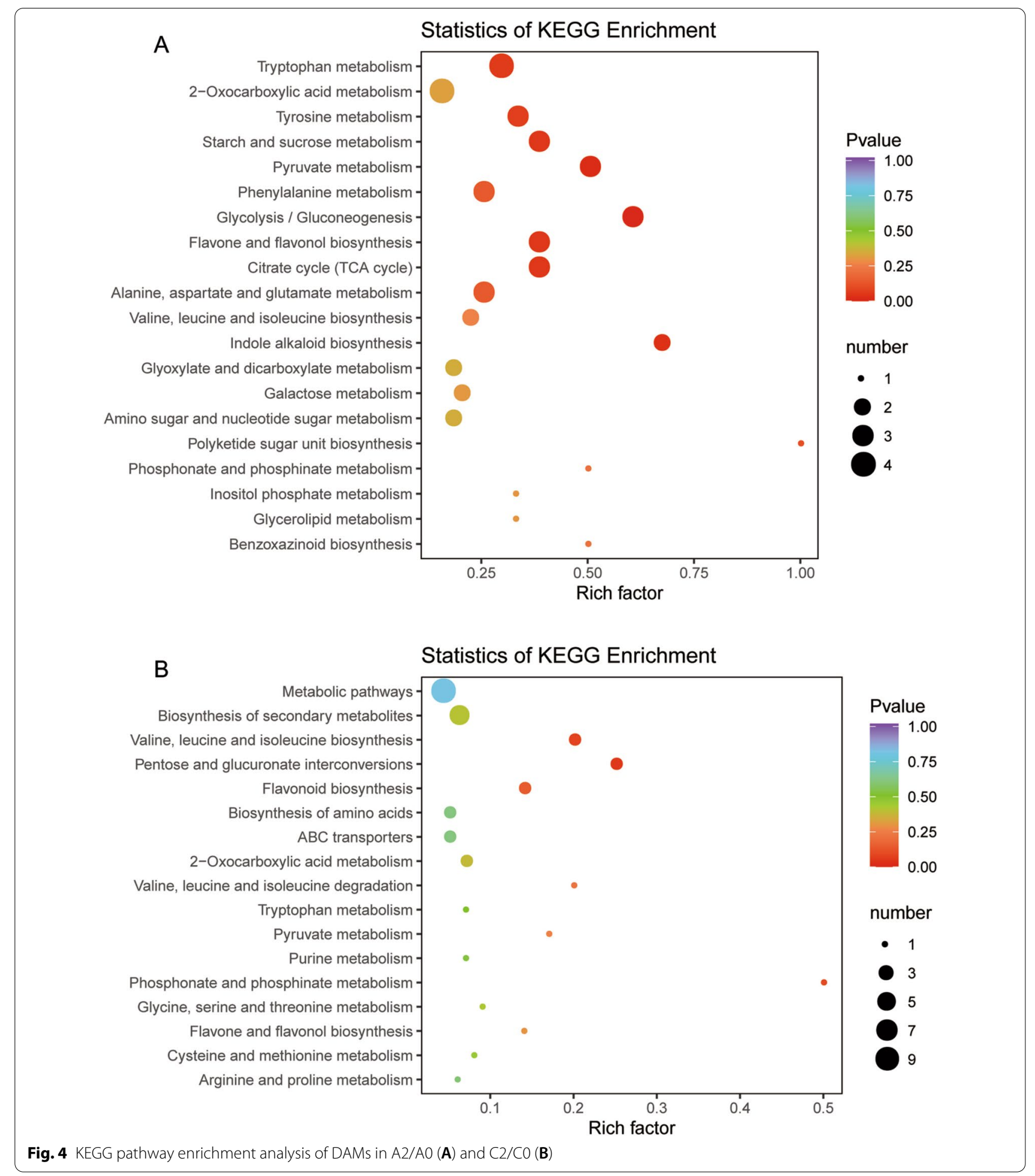

accumulation (418 DAMs and 39 co-DAMs) among the three natural genotypic maize lines were greater than those among GM/non-GM maize lines, with 330 DAMs and 3 co-DAMs observed.
Previous published proteomics data showed that the insertion of dual herbicide-resistance genes led to an increase in the number of DEPs, regardless of whether the genetic background was the same (A2/A0 compared 
A

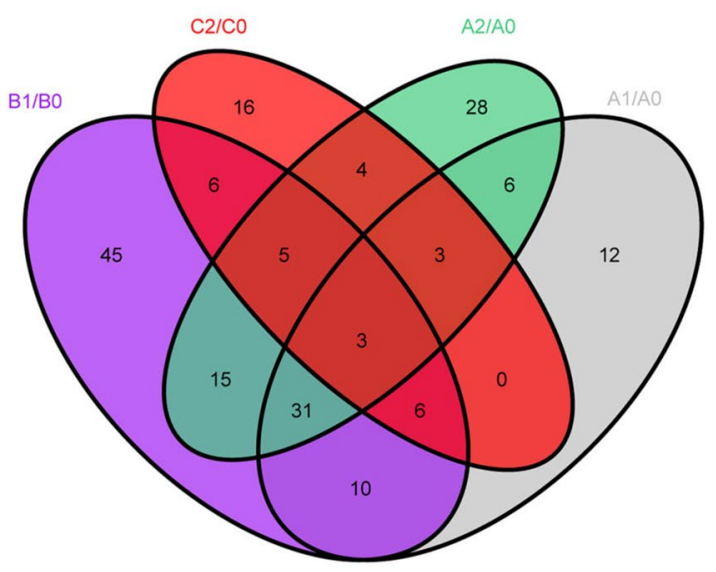

B

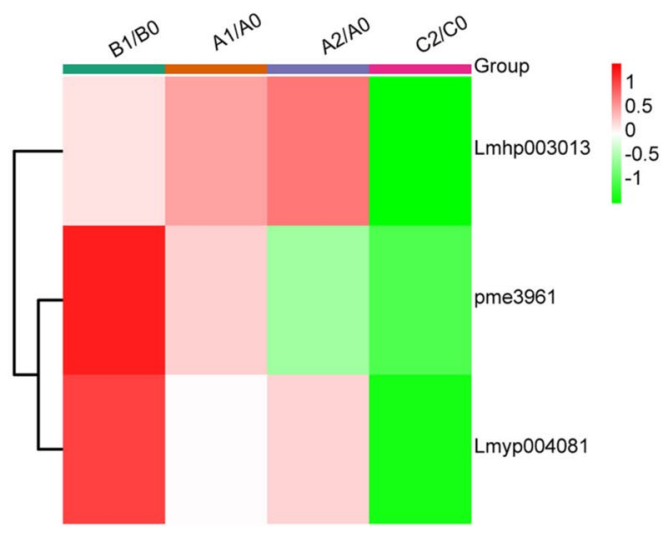

C

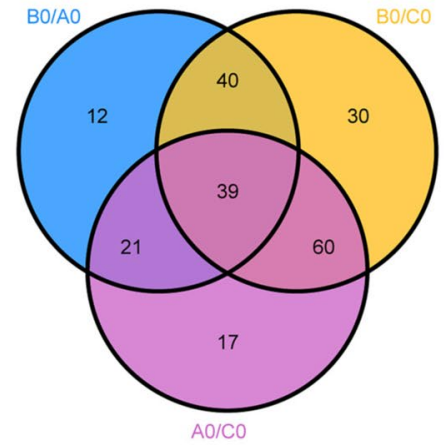

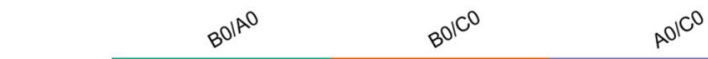

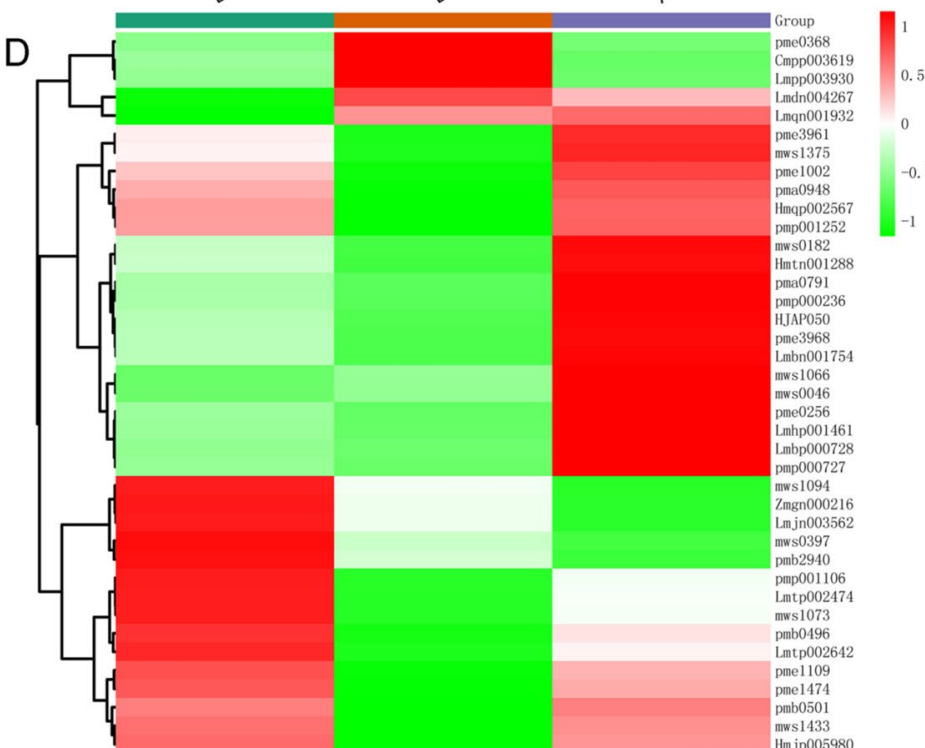

Fig. 5 Identified co-DAMs. A Venn diagram showing the number of overlapping DAMs among 4 pairwise comparison groups of GM/non-GM maize seeds. B Cluster map comparing the co-DAM regulatory patterns of 4 pairwise comparison groups of GM/non-GM maize seeds. C Venn diagram showing the number of overlapping DAMs among 3 pairwise comparison groups of non-GM/non-GM maize seeds. D Cluster map comparing the co-DAM regulatory patterns of 3 pairwise comparison groups of non-GM/non-GM maize seeds. Red indicates relatively higher abundance, green indicates relatively lower abundance, and white indicates the same levels in the two lines. All the MS data were normalized and then used in cluster analysis

Table 2 Pathways associated with co-DAMs among the 4 GM maize lines

\begin{tabular}{llll}
\hline Index & Compounds & cpd_ID & kegg_map \\
\hline Lmhp003013 & N-Feruloyl-cadaverine & - & - \\
pme3961 & 2'-Deoxyadenosine & C00559 & ko00230 (purine metabolism), \\
& & & ko01100 (metabolic pathways) \\
Lmyp004081 & Isovitexin-2"-O-rhamnoside & - & - \\
\hline
\end{tabular}


Table 3 Number of (co)pathways involving DEPs and DAMs based on integrated proteomics and metabolomics analyses

\begin{tabular}{llll}
\hline Comparison groups & \multicolumn{3}{l}{$\begin{array}{l}\text { No. of pathways in which DEPs and/or } \\
\text { DAMs are involved }\end{array}$} \\
\cline { 2 - 4 } & DEPs & DAMs & Both \\
\hline A1/A0 & 61 & 24 & 14 \\
B1/B0 & 33 & 38 & 14 \\
A2/A0 & 72 & 45 & 31 \\
C2/C0 & 72 & 18 & 13 \\
\hline
\end{tabular}

with $\mathrm{A} 1 / \mathrm{A} 0)$ or different (C2/C0 compared with $\mathrm{B} 1 / \mathrm{B} 0)$ [16]. However, the metabolomics data showed different regulation trends, and the insertion of dual herbicideresistance genes (epsps and pat) led to an increase in the number of DAMs only with the same genetic background (A2/A0 compared with A1/A0); however, it led to a significant decrease in the number of DAMs with different genetic backgrounds ( $\mathrm{C} 2 / \mathrm{C} 0$ compared with $\mathrm{B} 1 / \mathrm{B} 0)$. This difference might have been due to the insertion of different foreign genes or insertions at different positions in the genome.

The difference in the number of DAMs between GM maize lines with the same exogenous genes but different receptors-A1/A0 compared with B1/B0 (71 and 121 ) and A2/A0 compared with $\mathrm{C} 2 / \mathrm{C} 0$ (95 and 43) - was much higher than that between GM maize lines with identical receptor but different exogenous genes, such as A1/A0 compared with A2/A0 (71 and 95). These results showed that different genetic backgrounds affected the metabolite profiles more than gene modification itself, which was consistent with previously reported results [25].

\section{Conclusions}

Widely targeted metabolomics was successfully used to evaluate variations in the metabolite profiles of maize seeds caused by both transgenic modifications and natural genotypic differences. The variations in metabolite accumulation among the different natural genotypic maize lines were greater than those caused by transgenic modification. The DAMs were not identified as new unintended metabolites; only their abundance changed. The different genetic backgrounds affect metabolite profiles more than gene modification itself does.

\footnotetext{
Abbreviations

GM: Genetically modified; DAMs: Differentially accumulated metabolites; ISAAA: The International Service for the Acquisition of Agri-biotech Applications; iTRAQ: Isobaric tags with relative and absolute quantitation; Bt: Bacillus thuringiensis; EPSPS: 5-Enolpyruvate shikimic acid-3-phosphate synthase; PAT: Phosphinothricin acetyl transferase; UPLC: Ultra-performance liquid
}

chromatography; MS: Mass spectrum; ESI-QTRAP: Electron spray ionizationtriple quadrupole-linear ion trap; QC: Quality control; OPLS-DA: Orthogonal partial least squares-discriminant analysis; VIP: The variable importance in projection; PCA: Principal component analysis; KEGG: Kyoto Encyclopedia of Genes and Genomes.

\section{Supplementary Information}

The online version contains supplementary material available at https://doi. org/10.1186/s40538-021-00257-0.

Additional file 1: Figure S1. Venn diagram showing the number of overlapping DAMs across pairwise comparisons among 4 comparison groups of GM/all non-GM maize seeds. Figure S2. DEPs and DAMs involved in phenylalanine, tyrosine and tryptophan biosynthesis pathways in the A2/A0 comparison group. Red dots are upregulated metabolites, green dots are downregulated metabolites, pink rectangles are upregulated proteins, and yellow rectangles are downregulated proteins. Table S1. List of all identified metabolites in maize seeds. Table S2. The list of maize DAMs in A1/A0. Table S3. The list of maize DAMs in B1/B0. Table S4. The list of maize DAMs in A2/A0. Table S5. The list of maize DAMs in C2/C0. Table S6. The list of maize DAMs in A0/CO. Table S7. The list of maize DAMs in B0/CO. Table S8. The list of maize DAMs in B0/AO.

\section{Acknowledgements}

We are grateful to Dr. Haiyi Zhao (Genecreate Biological Engineering Co., Ltd. Wuhan, China) for technical assistance with the MS.

\section{Authors' contributions}

WL and WJ conceived and designed the experiments. WL, MD and LM performed the experiments. WL and YW analysed the data. WL and WJ wrote the paper. All authors reviewed the manuscript. All authors read and approved the final manuscript.

\section{Funding}

This work was supported by the 2020 Research Program of Sanya Yazhou Bay Science and Technology City (No. SKJC-2020-02-005) and the National Transgenic Major Program of China (No. 2019ZX08013011).

\section{Availability of data and materials}

All data generated or analysed during this study are included in this published article and its supplementary information files.

\section{Declarations}

Ethics approval and consent to participate Not applicable.

\section{Consent for publication}

Not applicable.

\section{Competing interests}

The authors declare that they have no competing interests.

Received: 19 July 2021 Accepted: 19 September 2021

Published online: 23 November 2021

References

1. Conner AJ, Jacobs JM. Food risks from transgenic crops in perspective. Nutrition. 2000;16(7-8):709-11.

2. Conner AJ, Jacobs JM. Genetic engineering of crops as potential source of genetic hazard in the human diet. Mutat Res. 1999;443(1-2):223-34.

3. Ren YF, et al. A comparative proteomics approach to detect unintended effects in transgenic Arabidopsis. J Genet Genomics. 2009;36(10):629-39.

4. Cellini F, et al. Unintended effects and their detection in genetically modified crops. Food Chem Toxicol. 2004;42(7):1089-125. 
5. Li X, et al. Comparative analysis of nutritional composition between herbicide-tolerant rice with bar gene and its non-transgenic counterpart. J Food Compos Anal. 2008;21(7):535-9.

6. Han JH, et al. Comparison of nutrient composition of parental rice and rice genetically modified with cowpea trypsin inhibitor in China. J Food Compos Anal. 2005;18(4):297-302.

7. Ricroch AE, Berge JB, Kuntz M. Evaluation of genetically engineered crops using transcriptomic, proteomic, and metabolomic profiling techniques. Plant Physiol. 2011;155(4):1752-61.

8. Barros $\mathrm{E}$, et al. Comparison of two GM maize varieties with a near-isogenic non-GM variety using transcriptomics, proteomics and metabolomics. Plant Biotechnol J. 2010;8(4):436-51.

9. Bedair M, Glenn KC. Evaluation of the use of untargeted metabolomics in the safety assessment of genetically modified crops. Metabolomics. 2020;16(10):1-5.

10. Qin J, et al. Proteomic analysis of elite soybean Jidou17 and its parents using iTRAQ-based quantitative approaches. Proteom Sci. 2013;11(1):12

11. Qin J, et al. iTRAQ protein profile analysis of developmental dynamics in soybean [Glycine max (L.) Merr.] leaves. PLoS ONE. 2017;12(9):e0181910.

12. Zeng WY, et al. Proteomic analysis by iTRAQ-MRM of soybean resistance to Lamprosema Indicate. BMC Genomics. 2017;18:1-22.

13. Ji W, et al. Comparative proteomic analysis of soybean leaves and roots by iTRAQ provides insights into response mechanisms to short-term salt stress. Front Plant Sci. 2016:7:573.

14. Lim S, et al. Proteomics analysis suggests broad functional changes in potato leaves triggered by phosphites and a complex indirect mode of action against Phytophthora infestans. J Proteomics. 2013;93:207-23.

15. Qian D, Tian L, Qu L. Proteomic analysis of endoplasmic reticulum stress responses in rice seeds. Sci Rep. 2015;5:14255.

16. Liu WX, et al. iTRAQ-based quantitative proteomic analysis of transgenic and nontransgenic maize seeds. J Food Compos Anal. 2020. https://doi. org/10.1016/j.jfca.2020.103564.

17. Schauer $\mathrm{N}$, et al. Comprehensive metabolic profiling and phenotyping of interspecific introgression lines for tomato improvement. Nat Biotechnol. 2006;24(4):447-54.

18. Schauer $\mathrm{N}$, et al. Mode of inheritance of primary metabolic traits in tomato. Plant Cell. 2008;20(3):509-23.

19. Alonso-Blanco $C$, et al. What has natural variation taught us about plant development, physiology, and adaptation? Plant Cell. 2009;21(7):1877-96

20. Chen $W$, et al. A novel integrated method for large-scale detection, identification, and quantification of widely targeted metabolites: application in the study of rice metabolomics. Mol Plant. 2013:6(6):1769-80.

21. Zhang $X$, et al. Negligible transcriptome and metabolome alterations in RNAi insecticidal maize against Monolepta hieroglyphica. Plant Cell Rep. 2020;39(11):1539-47.
22. Liu QS, et al. Plant breeding involving genetic engineering does not result in unacceptable unintended effects in rice relative to conventional cross-breeding. Plant J. 2020;103(6):2236-49.

23. Piccioni F, et al. NMR metabolic profiling of transgenic maize with the Cry1Ab gene. J Agric Food Chem. 2009;57(14):6041-9.

24. Bernal JL, et al. Use of supercritical fluid extraction and gas chromatography-mass spectrometry to obtain amino acid profiles from several genetically modified varieties of maize and soybean. J Chromatogr A. 2008;1192(2):266-72.

25. Fu W, et al. Evaluation on reprogramed biological processes in transgenic maize varieties using transcriptomics and metabolomics. Sci Rep. 2021;11(1):2050.

26. Chakroun M, et al. Bacterial Vegetative insecticidal proteins (Vip) from entomopathogenic bacteria. Microbiol Mol Biol Rev. 2016;80(2):329-50.

27. Arruda SC, et al. Comparative studies focusing on transgenic through cp4EPSPS gene and non-transgenic soybean plants: an analysis of protein species and enzymes. J Proteomics. 2013;93:107-16.

28. Garcia MJ, et al. The triple amino acid substitution TAP-IVS in the EPSPS gene confers high glyphosate resistance to the superweed Amaranthus hybridus. Int J Mol Sci. 2019;20(10):2396.

29. He M, et al. A new type of class I bacterial 5-enopyruvylshikimate-3-phosphate synthase mutants with enhanced tolerance to glyphosate. Biochim Biophys Acta. 2001;1568(1):1-6.

30. Wehrmann A, et al. The similarities of bar and pat gene products make them equally applicable for plant engineers. Nat Biotechnol. 1996;14(10):1274-8

31. Thompson CJ, et al. Characterization of the herbicide-resistance gene bar from Streptomyces hygroscopicus. EMBO J. 1987;6(9):2519-23.

32. Liu W, et al. Integrated proteomics and metabolomics analysis of transgenic and gene-stacked maize line seeds. GM Crops Food. 2021:12(1):361-75.

33. Yuan $\mathrm{H}$, et al. Time-course comparative metabolite profiling under osmotic stress in tolerant and sensitive Tibetan Hulless Barley. Biomed Res Int. 2018:2018:9415409.

34. Kanehisa M, Goto S. KEGG: kyoto encyclopedia of genes and genomes. Nucleic Acids Res. 2000:28(1):27-30.

35. Ogata H, et al. KEGG: kyoto encyclopedia of genes and genomes. Nucleic Acids Res. 1999:27(1):29-34

\section{Publisher's Note}

Springer Nature remains neutral with regard to jurisdictional claims in published maps and institutional affiliations.

\section{Submit your manuscript to a SpringerOpen ${ }^{\circ}$ journal and benefit from:}

- Convenient online submission

- Rigorous peer review

- Open access: articles freely available online

- High visibility within the field

Retaining the copyright to your article

Submit your next manuscript at springeropen.com 